\title{
A case of airborne occupational eczema with hydroxyisohexyl 3-cyclohexene carboxyaldhehyde in Benin
}

\section{Fabrice Akpadjan ${ }^{1,2}$, Mênonli Adjobimey ${ }^{3}$, Rose Mikponhoué ${ }^{3}$, Serge Adè 4 , Vikkey Hinson ${ }^{3}$, Paul Ayélo $^{3}$, Christiane Koudoukpo ${ }^{4,5}$, Félix Atadokpèdé ${ }^{1}$}

${ }^{1}$ Research and Teaching Unit in Dermatology-Venereology, FSS Cotonou, Benin, ${ }^{2}$ Dermatology-Venereology Department of the CNHU/HKM of Cotonou, Benin, ${ }^{3}$ Research and Teaching Unit in Occupational Health and Environment, FSS Cotonou, Benin, ${ }^{3}$ Research and Teaching Unit in Pneumology, Faculty of Medicine of Parakou; CHUD/B-A, Parakou, Benin, ${ }^{4}$ Research and Teaching Unit in Dermatology-Venereology, Faculty of Medicine of Parakou, Parakou, Benin, ${ }^{5}$ Dermatology-Venereology Department of the Borgou-Alibori CHUD, Parakou, Benin

Corresponding author: Dr. Fabrice Akpadjan, E-mail: barfice@yahoo.fr

\begin{abstract}
The existence of airborne contaminants in the professional environment can lead to allergic manifestations, especially in people with a history of allergies. We report a case of eczema with hydroxyisohexyl 3-cyclohexene carboxyaldhehyde (HICC), present in a patient's professional environment. This was a 53 -year-old patient, an archivist by training with more than 15 years' experience, with a history of asthma and facial eczema. She had two episodes of asthma attacks associated with eczema of the face and neckline in 6 months. The patch test performed with the European standard battery showed a bi-sensitization to Budesonide and Hydroxyisohexyl 3-cyclohexene carboxyaldhehyde. The patient was recommended to avoid the allergen in both professional and non-professional settings. Indirect occupational exposure should be considered in the event of acute allergic manifestations in workers who are usually not exposed and who show signs in the work environment.
\end{abstract}

Key words: Airborne eczema; Professional environment; Patch test; Hydroxyisohexyl 3-cyclohexene carboxyaldhehyde

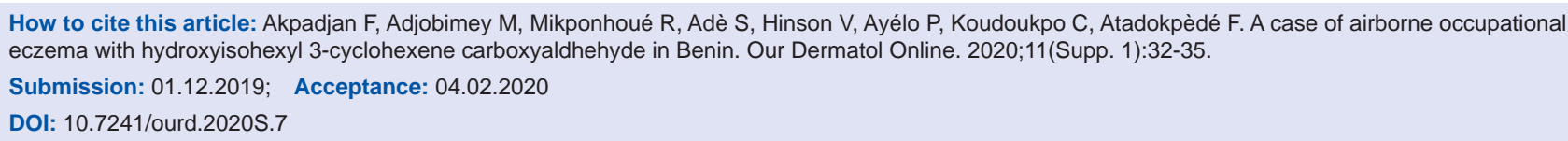




\section{Un cas d'eczéma professionnel aéroporté à l'hydroxyisohexyl 3-cyclohexene carboxyaldhéhyde au Bénin}

\section{Fabrice Akpadjan ${ }^{1,2}$, Mênonli Adjobimey ${ }^{3}$, Rose Mikponhoué ${ }^{3}$, Serge Adè ${ }^{4}$, Vikkey Hinson ${ }^{3}$, Paul Ayélo $^{3}$, Christiane Koudoukpo ${ }^{4,5}$, Félix Atadokpèdé ${ }^{1}$}

${ }^{1}$ Research and Teaching Unit in Dermatology-Venereology, FSS Cotonou, Benin, ${ }^{2}$ Dermatology-Venereology Department of
the CNHU/HKM of Cotonou, Benin, ${ }^{3}$ Research and Teaching Unit in Occupational Health and Environment, FSS Cotonou,
Benin, ${ }^{3}$ Research and Teaching Unit in Pneumology, Faculty of Medicine of Parakou; CHUD/B-A, Parakou, Benin,
${ }^{4}$ Research and Teaching Unit in Dermatology-Venereology, Faculty of Medicine of Parakou, Parakou, Benin,
${ }^{5}$ Dermatology-Venereology Department of the Borgou-Alibori CHUD, Parakou, Benin

Corresponding author: Dr. Fabrice Akpadjan, E-mail: barfice@yahoo.fr

\section{RÉSUMÉ}

Lexistence d'aéro-contaminants dans l'environnement professionnel peut entrainer des manifestations allergiques surtout chez les sujets aux antécédents allergiques. Nous rapportons un cas d'eczéma à l'hydroxyisohexyl 3-cyclohexene carboxyaldhéhyde (HICC) ou Lyral ${ }^{\circledR}$, présent dans l'environnement professionnel d'une patiente. Il s'est agi d'une patiente de 53 ans, archiviste de formation avec plus de 15 ans d'ancienneté, aux antécédents d'asthme et d'eczéma du visage. Elle a présenté en 6 mois d'intervalle deux épisodes de crises d'asthme associé à un eczéma du visage et du décolleté. Le patch test réalisé avec la batterie standard européenne a montré une bi-sensibilisation au Budésonide et à l'Hydroxyisohexyl 3-cyclohexene carboxyaldhehyde $\left(\mathrm{Lyral}^{\circledR}\right)$. Une éviction de l'allergène aussi bien en milieu professionnel que non professionnel a été recommandée à la patiente. Une exposition professionnelle indirecte est à évoquer en cas de manifestations allergiques aigues chez des travailleurs non exposés habituellement, présentant des signes sur les lieux de travail.

Mots clés: Eczéma aéroporté; Environnement professionnel; Patch test; Lyral ${ }^{\circledast}$

\section{INTRODUCTION}

Leczéma de contact aux parfums est fréquent, il siège en règle au visage, au cou et parfois au pli axillaire ou aux mains. Il peut être aéroporté, sous forme d'aérosol. Lallergie de contact aux parfums est la cause la plus fréquente des dermatites de contact dues aux cosmétiques [1]. Le premier mélange de parfums à $8 \%$ (Fragrance Mix I) utilisé dans la batterie standard européenne ne permettait pas d'identifier tous les patients ayant des antécédents positifs de réactions indésirables aux parfums. Ainsi un deuxième mélange de parfums (Fragrance Mix II), avec 6 produits chimiques fréquemment utilisés a été constitué. Il a été testé en 3 concentrations: $28 \%$,
$14 \%$ et $10 \%$. Mais la concentration moyenne de 14\% de Fragrance Mix II, semble être le meilleur outil de diagnostic. Il contient entre autre 2,5\% d'hydroxyisohexyl 3-cyclohexène carboxaldéhyde [2] Depuis 2008, il est donc recommandé d'effectuer des tests épicutanés supplémentaires avec 5\% d'hydroxyisohexyl 3-cyclohexène carboxaldéhyde et la fragrance mix II [3].

La plupart des gens dans la société moderne sont exposés quotidiennement à des ingrédients parfumants provenant d'une ou de plusieurs sources [4]. Ainsi les patients allergiques aux ingrédients de parfums peuvent être directement ou indirectement exposés, aussi bien dans leur vie privée que professionnelle.

\footnotetext{
How to cite this article: Akpadjan F, Adjobimey M, Mikponhoué R, Adè S, Hinson V, Ayélo P, Koudoukpo C, Atadokpèdé F. Un cas d'eczéma professionnel aéroporté à l'hydroxyisohexyl 3-cyclohexene carboxyaldhéhyde au Bénin. Our Dermatol Online. 2020;11(Supp. 1):32-35.

Submission: 01.12.2019; Acceptance: 04.02.2020

DOI: $10.7241 /$ ourd.2020S.7
} 
Nous rapportons un cas d'eczéma à l'hydroxyisohexyl 3-cyclohexane carboxyaldhéhyde, présent dans l'environnement professionnel d'une patiente.

\section{OBSERVATION}

Il s'est agi d'une patiente de 53 ans, archiviste de formation, ayant une ancienneté de plus de 15 ans. Elle a un antécédent de crise d'asthme et d'eczéma du visage il y a 10 ans, survenus sur les lieux du travail. Elle a présenté en 6 mois d'intervalle en 2018 deux épisodes de crises d'asthme sévère associé à un eczéma du visage et du décolleté ayant nécessité une consultation aux urgences puis successivement en pneumologie, en dermatologie, en allergologie et en médecine du travail. Le premier épisode était survenu un lundi matin alors que les locaux du service avaient été badigeonnés le weekend. Le deuxième épisode était survenu après pulvérisation de désodorisant par un collègue de service à un poste de travail voisin. Le patch test réalisé avec la batterie standard européenne (Fig. 1) a montré une bi-sensibilisation au Budésonide et à l'Hydroxyisohexyl 3-cyclohexane carboxyaldhehyde $\left(\right.$ Lyral $\left.^{\circledR}\right)$ alors que le Fragrance mix II et la methylisothiazolinone étaient négatifs.

Le prick test aux pneumallergènes (Dermatophagoides ptéronyssinus Dermatophagoides farinae, Blomia tropicalis, Alternaria alternata, Chien, Chat) était aussi négatif (Fig. 2). Une prise en charge multidisciplinaire avait permis de contrôler chaque crise; elle était basée sur une corticothérapie locale et orale, les Beta-2mimétiques et les antihistaminiques. Une éviction de l'allergène a été préconisée au travailleur en milieu professionnel ou non, afin de prévenir de nouvelles crises.

\section{DISCUSSION}

La dermatite de contact aéroportée (DCA) est une pathologie fréquente, et l'origine professionnelle de la dermatite de contact aéroportée est de plus en plus reconnue [5]. Le Lyral ${ }^{\circledR}$ fait partie des 26 substances allergènes réglementées par l'Europe. Il est principalement utilisé dans les parfums pour ses senteurs florales. Il est présent dans la parfumerie, la peinture, les produits d'ambiance et les produits d'entretien. Il est inclus dans le mélange de parfums (fragrance mix 2) à 2,5\%. Malgré cette inclusion le fragrance mix 2 testé chez cette patiente était négative. Mais le Lyral ${ }^{\circledR}$ isolé à $5 \%$ était positive. On peut donc

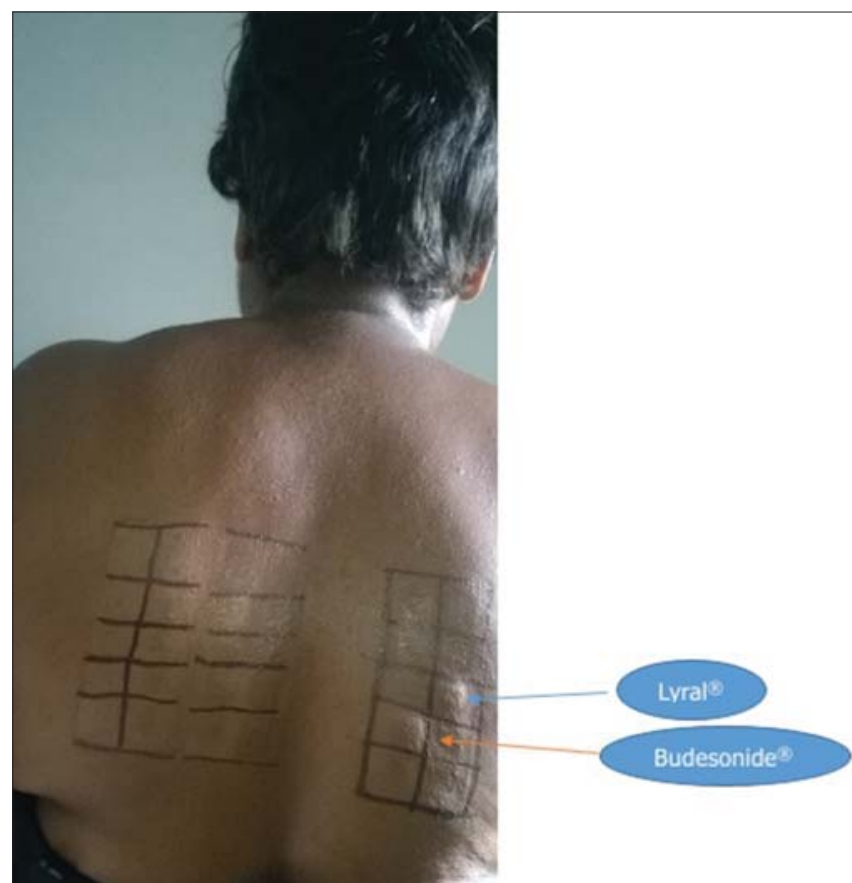

Figure 1: Lecture à $48 \mathrm{~h}$ du patch test réalisé avec la batterie standard européenne, montrant une bi-sensibilisation au Budésonide et à l'Hydroxyisohexyl 3-cyclohexène carboxyaldhéhyde (Reading at 48 hours of the patch test performed with the European standard battery, showing a bi-sensitization to Budesonide and Hydroxyisohexyl 3-cyclohexene carboxyaldhehyde).

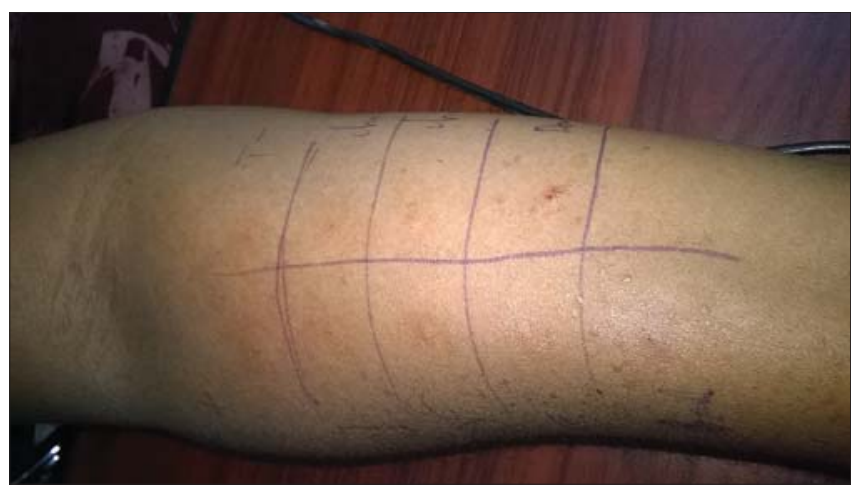

Figure 2: Négativité du prick test réalisé sur la face antérieure de l'avant-bras droit (Negativity of the prick test performed on the anterior surface of the right forearm).

déduire que seul, et à fort pourcentage le Lyral ${ }^{\circledR}$ est plus allergisant. Dans une étude allemande réalisée par Geier et al., les parfums les plus importants dans les fragrances mix étaient l'absolue de mousse de chêne et l'hydroxyisohexyl 3-cyclohexène carboxaldéhyde [6] Dans une autre étude coréenne, parmi les fragrances spécifiques, le Lyral ${ }^{\circledast}$ (hydroxyisohexyl 3-cyclohexane carboxdaldéhyde) faisait partie des allergènes montrant le plus souvent une forte positivité [1].

De rares cas d'eczémas professionnels liés au Lyral ${ }^{\circledR}$ ont été signalés dans la littérature. Un cas de sensibilisation 
au Lyral ${ }^{\circledR}$ a été constaté chez une esthéticienne qui a manipulé plusieurs produits cosmétiques, tant dans son travail que dans sa vie privée, et qui a présenté un eczéma de contact des mains avec des périodes d'extension aux membres supérieurs et au tronc [7].

La particularité chez notre patiente est le caractère indirect de son exposition au $\mathrm{Lyral}^{\circledR}$ sur son lieu de travail et surtout de sources différentes (peinture et déodorant).

Dans une étude réalisée au Danemark en 2012 la prévalence de la dermatite de contact allergique causée par l'hydroxyisohexyl 3-cyclohexène carboxaldéhyde n'a pas changé sur une période de neuf ans; et le Lyral ${ }^{\circledR}$ restait une cause d'allergie de contact très pertinente et fréquente. Les nombreuses tentatives de l'IFRA (International Fragrance Association) pour réduire l'exposition au HICC dans les produits cosmétiques ne semblaient pas être suffisantes pour réduire l'allergie aux HICC [8]. Mais depuis 2017 le Lyral ${ }^{\circledR}$ est progressivement interdit en Europe; et à partir du 23 août 2021, son interdiction totale sera prononcée [9].

Parmi les allergènes responsables d'eczéma professionnel aéroporté, c'est plutôt la méthylisothiazolinone (MI) qui vient en tête; c'est un agent de conservation largement utilisé dans les produits de consommation et les produits industriels. Elle est une cause bien connue de dermatite de contact allergique dans le monde entier. Son exposition aéroportée dans la peinture est un risque professionnel reconnu [10]. Mais le patch test réalisé chez notre patiente a montré une négativité à la methylisothiazolinone.

\section{CONCLUSION}

Leczéma professionnel aéroporté à l'hydroxyisohexyl 3-cyclohexène carboxyaldhéhyde est rarement décrit dans la littérature. Le Lyral ${ }^{\circledR}$ est le plus souvent impliqué dans les allergies de contact par exposition directe chez les femmes sensibilisées et qui utilisent des cosmétiques le contenant. Une exposition professionnelle indirecte est à évoquer en cas de manifestations allergiques aiguës chez des travailleurs non exposés habituellement, présentant des signes sur les lieux de travail. Il s'agit du premier cas documenté d'eczéma professionnel au Lyral ${ }^{\circledR}$ observé au Bénin.

\section{Consent}

The examination of the patient was conducted according to the Declaration of Helsinki principles.

The authors certify that they have obtained all appropriate patient consent forms. In the form the patient(s) has/have given his/her/ their consent for his/her/their images and other clinical information to be reported in the journal. The patients understand that their names and initials will not be published and due efforts will be made to conceal their identity, but anonymity cannot be guaranteed.

\section{RÉFÉRENCES}

1. An S, Lee AY, Lee CH, Kim DW, Hahm JH, Kim KJ, et al. Fragrance contact dermatitis in Korea: A joint study. Contact Dermatitis. 2005;53:320-3.

2. Frosch PJ, Pirker C, Rastogi SC, Andersen KE, Bruze M, Svedman C, et al. Patch testing with a new fragrance mix detects additional patients sensitive to perfumes and missed by the current fragrance mix. Contact Dermatitis. 2005;52:207-15.

3. Bruze M, Andersen KE, Goossens A. Recommendation to include fragrance mix 2 and hydroxyisohexyl 3-cyclohexene carboxaldehyde $\left(\mathrm{Lyral}^{\mathbb{R}}\right)$ in the European baseline patch test series. Contact Dermatitis. 2008;58:129-33.

4. Johansen JD. Fragrance Contact Allergy: A Clinical Review. Am J Clin Dermatol. 2003;4:789-98.

5. Tončić RJ, Balić A, Pavičić B, Žužul K, Petković M, Bartolić L, et al. Occupational airborne contact dermatitis caused by omeprazole. Acta Dermatovenerologica Croat. 2019;27:188-9.

6. Geier J, Uter W, Lessmann H, Schnuch A. Aktuelle Kontaktallergene. Hautarzt. 2011;62:751-6.

7. Heras Mendaza F, Díaz-Recuero JL, Cabello MJ, Conde-Salazar L. Sensibilización al Lyral ${ }^{\circledR}$. Actas Dermosifiliogr. 2006;97:374-8.

8. Heisterberg M V., Laurberg G, Veien NK, Menné T, Avnstorp C, Kaaber K, et al. Prevalence of allergic contact dermatitis caused by hydroxyisohexyl 3-cyclohexene carboxaldehyde has not changed in Denmark. Contact Dermatitis. 2012;67:49-51.

9. Institut HYSOPE France. LYRAL ${ }^{\circledR}$ : interdiction effective en Europe. [En ligne] https://www.institut-hysope.com/new/actualites/lyralinterdiction-effective-en-europe/. Consulté le 30 novembre 2019.

10. Wright AM, Cahill JL. Airborne exposure to methylisothiazolinone in paint causing allergic contact dermatitis: An Australian perspective. Australas J Dermatol. 2016;57:294-5.

Copyright by Fabrice Akpadjan, et al. This is an open access article distributed under the terms of the Creative Commons Attribution License, which permits unrestricted use, distribution, and reproduction in any medium, provided the original author and source are credited.

Source of Support: Nil, Conflict of Interest: None declared. 